\title{
Analysis of the Impacts of Cochlear Implants in the Adults of Sensorineural Hearing Loss: What Is New and Not So New: A Literature Review
}

\author{
Sohail Ahmed a, Haroon Akram ${ }^{\mathrm{a}}$, Prof. Yonghua Wang ${ }^{*}$, Xiaohui Yan ${ }^{\mathrm{b}}$, Chenlu Wang ${ }^{\mathrm{b}}$, Prof. Wendi Shi* \\ ${ }^{a}$ Zhejiang Chinese medical university \\ ${ }^{\mathrm{b}}$ Hangzhou Ren-ai hearing rehabilitation center, China \\ *Correspondence: \\ Prof. Yonghua Wang, Zhejiang Chinese Medical University, Hangzhou, 310053, Zhejiang Province, China \\ Prof. Wendi Shi, Hangzhou Ren-ai Hearing Rehabilitation Research Centre, Hangzhou, China
}

\begin{abstract}
PURPOSE: the purpose of this systematic review is to advise on advantages and clinical outcomes of Cochlear implants in adult population with sensorineural hearing loss.

Methods: A systematic search of several electronic databases, including PubMed and Embase, was used to identify relevant studies for inclusion.

RESULTS: 16 articles were included in our study most of the studies showed better speech recognition, quality of life, speech perception and social interactions. Consistent use of implant showed better results as well as early implantation.

\section{CONCLUSION:}

Early intervention and consistent use of implant is necessary for desired outcome. Sensorineural hearing loss have many different causes from simple infection to genetic mutations.
\end{abstract}

DOI: $10.7176 / \mathrm{JHMN} / 93-05$

Publication date:September $30^{\text {th }} 2021$

\section{INTRODUCTION}

The prevalence of hearing loss increases with age. In people aged over 65 years it is $30-60 \%$, and increases to $70-90 \%$ in people aged over 85 years $^{1-3}$. In older adults (i.e., $>65$ years) the negative impact of hearing loss on quality of life is substantial, with population norms showing that any form of hearing disability results in poorer physical and mental health outcomes. People with a greater degree of hearing loss are the most affected ${ }^{4-6}$. The cochlear implantation represents the gold standard technique to restore the hearing in deafened patients. The cochlear implant is able to replace the function of hair cells that are no longer able to generate electrical impulses in response to sound, representing a bionic organ able to bypass the transduction mechanism of the sound wave normally done in the outer, middle and inner ear and directly stimulate the spiral ganglion of the cochlear nerve. Nevertheless, despite the overall excellent results in speech perception among cochlear implanted patients, results are still heterogeneous with some implanted patients being poor performers after unilateral and/or bilateral cochlear implantation ${ }^{7}$. Moreover some improvements still have to be done in the processing of signal and the stimulation strategy in order to improve the speech understanding in difficulty noisy condition and quality of the perceived sound (e.g. appreciation of music). The classical indication for cochlear implantation was the severe to profound sensorineural hearing loss; over the past decade indications for cochlear implantation evolved and include now also the hearing loss involving only high frequencies or in some selected cases of single sided deafness. As a consequence, surgery has evolved toward a low intra-cochlear trauma insertion in order to maintain the integrity of inner ear structures in all cochlear implants recipients, even for those destined to electric-only stimulation. Minimizing trauma during implantation may offer several advantages. For patients with "usable" pre-implant low frequency hearing, limiting trauma can allow for the preservation of the residual hearing, allowing the electric-acoustic stimulation. For all the other patients, reducing intra-cochlear damage may limit the fibrosis and ossification, making easier the revision surgery for device failure or upgrade; this is becoming increasingly important as more patients are undergoing implantation during infancy and early childhood. Moreover, limiting injury potentially allows for the application of future technologies, such as cellular regeneration or other novel cochlear nerve stimulation technologies ${ }^{8}$

Types of hearing loss Based on the hearing measurements, hearing loss can be conductive, sensorineural and mixed. In the sensorineural form, either lesion of the inner ear (cochlear) or the hearing pathway (retrocochlear) can occur. Etiology of sensorineural hearing loss Sensori-neural hearing loss can be present even at birth or may develop during later stages of life. The cause of congenital sensorineural hearing loss remains unclear in $57 \%$, with $18 \%$ of acquired origin and $25 \%$ with genetic background. Usually, functional failure of the hair cells can be found in these patients, but in $20 \%$ of the cases, bony malformation of the cochlea can be detected as well. Hearing loss due to environmental factors can develop in any stage of life. Among these factors, 
usage of drugs with ototoxic side effects like aminoglycoside antibiotics (kanamycin, gentamicin) or diuretics (furosemide, ethacrynic acid) is very common. Degree of hearing loss Hearing loss can also be classified according to its severity. Mild, moderate, severe and profound hearing loss as well as complete deafness can be distinguished. The type of rehabilitation is determined by the degree of hearing loss. The way of hearing rehabilitation is determinate by the type or degree of the hearing loss. Hearing aid is the most frequently used method in sensorineural hearing loss, amplifying the signal to replace the non-functional outer hair cells. Furthermore, implantable hearing devices are also available on the market. ${ }^{9}$

The purpose of our review is to go through the data available in order to understand the clinical, social, educational and professional outcomes of cochlear implants in adult patients with sensorineural hearing loss.

\section{METHODOLOGY:}

A literature review was planned and performed using methods specified in the Preferred Reporting Items for Systematic Reviews and Meta-Analyses (PRISMA) guidelines [23]. Initial search terms were compiled and iteratively refined by content experts in the fields of Otology, Neurotology, \& Cranial Base Surgery; Speech, Language, and Hearing Sciences; Library Science and Geriatrics. Both controlled vocabulary terms (e.g. MeSH) and key words were used to search the following databases for articles related to cochlear implants, sensorineural hearing loss, clinical outcomes, and adults: PubMed/ MEDLINE (1990-2021), Wiley/Cochrane Library (18982021), Thomson-Reuters/Web of Science (1898-2021) EBSCO/PsycINFO (1880's-present), and EBSCO/CINAHL (1981-2021) Inclusion criteria were

- Population: the study had to include adult individuals

- Intervention with cochlear implantion (i.e. studies that looked only at hearing aids were excluded).

\section{SYSTEMATIC REVIEWS:}

Oliver et al conducted a study on twenty eight patients, older than 60 years and with profound bilateral sensorineural hearing loss, received a cochlear implant between 1991 and 2001 . The mean age was 66 years and the median follow-up was 22.5 months. Speech perception scores before and after implantations were analyzed retrospectively in order to evaluate the benefit of cochlear implantation. All patients significantly improved their speech performances. The mean preoperative disyllabic words score in best aided conditions (hearing aids and lipreading) was $529 / 27 \%$, and this improved to a post-implantation mean of $839 / 23 \%(\mathrm{p} / 0.0094)$. The mean preoperative sentences score in best aided conditions (hearing aids and lipreading) was 679/ 34\%, and this improved to a post-implantation mean of $919 / 20 \%$ (p/0.0032). Patients over 70 years old performed as well as those who are younger the surgical procedure was well tolerated in all patients. One patient developed a postoperative vertigo due to a peri-lymphatic fistula, which required a revision procedure to better close the cochleostomy. There was a device failure 7 years after implantation with a Laura ABS device. Similar benefit was obtained 6 months after reimplantation with a Nucleus 24- channel device. Among the 28 patients, one died 4 years after surgery (cancer). An alteration of intellectual abilities was reported in two patients. One of them became a non-user. Twenty-five patients used the device for more than $12 \mathrm{~h}$ per day. Thirteen patients used the telephone. $^{10}$

Angeli et al conducted a prospective cohort study on 60 participants to determine the etiology of adult-onset sensorineural hearing loss. 60 adult subjects with bilateral sensorineural hearing loss of no obvious etiology by medical history and physical examination. These patients were evaluated at an academic medical center and underwent evaluation by high-resolution computed tomography of the temporal bone, autoimmune panel, and DNA testing for mutations of both the GJB2 gene and the mitochondrial DNA (1555A $>\mathrm{G}$ and $7445 \mathrm{~A}>\mathrm{G})$. An etiologic diagnosis was achieved in 6 patients: cochlear otosclerosis, 1 case; dilated vestibular aqueduct, 1 case; a mitochondrial DNA 7445A $>$ G mutation, 3 cases; and a mitochondrial DNA 1555A $>\mathrm{G}$ mutation, 1 case. This result underscores the importance of a search for the etiology of a hearing deficit in adult patients. There are specific interventions now available for the management of hearing-impaired patients with cochlear otosclerosis and mitochondrial DNA mutations. ${ }^{11}$

Based on pre-implantation audiometric thresholds and aided speech recognition scores, the data of $164 \mathrm{CI}$ recipients were divided into a group of patients that fulfilled conservative criteria (mean hearing loss at $0.5,1$ and $2 \mathrm{kHz}>85 \mathrm{~dB}$ HL and phoneme scores with hearing aids $<30 \%$ ), and the remaining group of patients that felt outside this conservative criterion. Speech recognition scores (in quiet) and quality of life (using the NCIQ) of both groups, measured at 1-year post-implantation, were compared. The group that felt outside the conservative criterion showed a higher phoneme score at 1-year post-implantation compared to the conservative group, suggesting that relaxed criteria have a positive influence on the speech recognition results with CI. With respect to quality of life, both groups significantly improved 1-year post-implantation. The conservative group showed a higher benefit on the advanced perception domain of the NCIQ. Based on their worse pre-implantation hearing, this was expected. The data suggest that relaxation of CI indication positively affects the speech recognition performance of patients with severe hearing loss. Both groups of patients showed a positive effect of 
CI on the quality of life. This benefit relates to communication skills and the subjective day-to-day functioning in society. ${ }^{12}$

Study conducted on Twenty-five patients treated for severe hearing loss after blunt head trauma. Patients assessed for cochlear implant candidacy criteria and post-implant outcomes in patients presenting with severe hearing loss after major blunt head trauma. Cause of injury, pure tone audiology, open set speech perception (preimplant and postimplant), promontory stimulation, and imaging results. Of the 25 patients with severe hearing loss, 11 ultimately underwent cochlear implantation. Fall from a height was the most common cause of injury. Five patients experienced bilateral temporal bone fractures. Seventeen patients satisfied candidacy criteria for implantation. For those patients who did undergo cochlear implantation, the mean post-implant open set speech recognition score was 71. Issues related to cochlear implant candidacy and outcomes for implantation will be discussed. Severe hearing loss after major blunt head trauma presents a number of unique clinical challenges. A significant number of these patients are not suitable for cochlear implantation for a variety of reasons. Clinicians working in the area of cochlear implantation should be aware of the various problems that can impact on effective cochlear implantation in such patients. ${ }^{13}$

A study conducted in 2018 on total of 45 adult cochlear implant recipients with asymmetric sensorineural hearing loss where performance for the best-aided condition exceeded $60 \%$ correct open set sentence recognition in quiet, and the implanted ear met traditional candidacy criteria. End point testing of the implanted ear was evaluated with use of the Consonant-Vowel Nucleus-Consonant (CNC) word test and AzBio sentence test materials in quiet, and bimodally with the AzBio sentence test materials in noise at $+5 \mathrm{~dB}$ signal-to-noise ratio (SNR). HRQoL was measured using the Nijmegen Cochlear Implant Questionnaire (NCIQ). Measured in quiet, with the non-implanted ear plugged, the average $\mathrm{CNC}$ word scores increased from $9.1 \%$ preoperatively to $55.7 \%$ $(p<0.01)$ at the 6-month post-activation test interval. Similarly, average AzBio sentence scores in quiet, with the non-implanted ear plugged, increased from $13.9 \%$ preoperatively to $73.4 \%(p<0.01)$ at the 6 -month postactivation test interval. Finally, in the bilateral/bimodal condition, the AzBio sentence score in $+5 \mathrm{~dB}$ SNR improved from an average of $26.8 \%$ preoperatively to $52.4 \%(p<0.01)$ at the 6 -month test interval. Results of the NCIQ showed improved scores on all six subdomains. These data demonstrate significant benefit of cochlear implantation among a group of postlingually deafened adults whose preoperative hearing and aided speech candidacy guidelines. Results of this study support the evaluation of a candidate's speech recognition in noise in the best-aided condition to adequately assess candidacy for a cochlear implant. ${ }^{14}$

A retrospective review on was performed comparing patients implanted at a younger (21 - 64 years) and older (65 and above) age. A total of 240 patients were evaluated. Patients experienced a significant improvement in audiologic performance as seen with word recognition scores $(\mathrm{p}<0.00001)$. The mean post-implant score was $44.6 \%$ (at 3 months) and $53.5 \%$ (at 24 months) at $50 \mathrm{~dB}$ compared to average pre-implantation aided score of $6 \%$. There was no significant difference between postoperative performances in younger versus older patient groups. Multiple regressions showed no correlation with duration of deafness at time of implantation or age and performance. There was no significant difference in performance based on side of implantation. This is one of the largest series to date on hearing outcomes in adults who receive a cochlear implant. No statistical differences were noted between the younger and older groups or based on side of implantation. The audiologic benefit in the adult population is clearly demonstrated. ${ }^{15}$

A FDA clinical trial was carried out to evaluate the potential benefit of cochlear implant (CI) use for adults with unilateral moderate-to-profound sensorineural hearing loss. Subjects were 20 adults with moderate-toprofound unilateral sensorineural hearing loss and normal or near-normal hearing on the other side. A MED-EL standard electrode was implanted in the impaired ear. Outcome measures included: (a) sound localization on the horizontal plane (11 positions, $-90^{\circ}$ to $90^{\circ}$ ), (b) word recognition in quiet with the CI alone, and (c) masked sentence recognition with the target at $0^{\circ}$ and the masker at $-90^{\circ}, 0^{\circ}$, or $90^{\circ}$. This battery was completed preoperatively and at $1,3,6,9$, and 12 months after CI activation. Normative data were also collected for 20 agematched control subjects with normal or near-normal hearing bilaterally. The CI improved localization accuracy and reduced side bias. Word recognition with the CI alone was similar to performance of traditional CI recipients. The CI improved masked sentence recognition when the masker was presented from the front or from the side of normal or near-normal hearing. The binaural benefits observed with the CI increased between the 1- and 3month intervals but appeared stable thereafter. In contrast to previous reports on localization and speech perception in patients with unilateral sensorineural hearing loss, CI benefits were consistently observed across individual subjects, and performance was at asymptote by the 3-month test interval. Cochlear implant settings, consistent CI use, and short duration of deafness could play a role in this result. ${ }^{16}$

Huarte et al conducted a study to determine whether individuals older than 60 years of age suffering from profound, bilateral, sensorineural hearing loss and unable to use hearing aids can benefit from a cochlear implant in order to improve hearing performance. Additionally, to evaluate how they manage the external components of their implanted system. The records of 68 cochlear implant users over 60 years of age were reviewed. They had undergone tonal and speech audiometry evaluations of hearing before and after implantation. Subsets of 27 
elderly patients were asked to respond to a specially designed questionnaire regarding the use of the external components of the implanted system. Patients over 60 years of age benefit from cochlear implants. The earlier the implantation, the better the results tend to be. Certain limitations when using the external components of the implanted system were evident among the elderly. People $>60$ years of age suffering from profound, bilateral, sensorineural hearing loss show improved hearing performance following cochlear implantation. Using the implanted system includes some limitations that should be studied and overcome. ${ }^{17}$

A prospective longitudinal study was conducted in tertiary referral center on seven adults with asymmetric sensorineural hearing loss, i.e., less than $30 \%$ aided speech recognition in their worst hearing ear and 60 to $85 \%$ speech recognition in their best hearing ear. All patients had a postlingual onset of their hearing loss and less than 20 years of auditory deprivation of their worst hearing ear. Cochlear implantation was fitted in the functionally deaf ear. Speech recognition in quiet, speech recognition in noise, spatial speech recognition, localization abilities, music appreciation, and quality of life. Measurements were performed before cochlear implantation and 3,6, and 12 months after cochlear implantation. Before cochlear implantation, the average speech recognition of the ear fitted with a hearing aid was $74 \%$. Cochlear implantation eventually resulted in average speech recognition of $75 \%$. Bimodal stimulation yielded speech recognition scores of 82,86 , and $88 \%$ after 3, 6, and 12 months, respectively. At all time intervals, bimodal stimulation resulted in significantly better speech recognition as compared with stimulation with only hearing aid or only cochlear implant (CI). Speech recognition in noise and spatial speech recognition significantly improved as well as the ability to localize sounds and the quality of life. This study demonstrated that patients are able to successfully integrate electrical stimulation with contralateral acoustic amplification and benefit from bimodal stimulation. Therefore, we think that cochlear implantation should be considered in this particular group of patients, even in the presence of substantial residual hearing on the contralateral side. ${ }^{18}$

The objective of Oliver et al study was to evaluate the benefit of cochlear implantation in adults aged 60 years and over. Twenty-eight patients, older than 60 years and with profound bilateral sensorineural hearing loss, received a cochlear implant between 1991 and 2001. The mean age was 66 years and the median follow-up was 22.5 months. Speech perception scores before and after implantation was analyzed retrospectively in order to evaluate the benefit of cochlear implantation. There was a significant improvement of the disyllabic words and sentences scores after implantation. The patients who were over 70 years performed as well as those who were younger. The surgical procedure was well tolerated in all patients. One patient developed a postoperative vertigo due to a perilymphatic fistula. In conclusion, cochlear implantation offers improvement in speech perception to the elderly population, as in the younger population. A careful assessment of the physical status of these patients remains essential in order to evaluate the risk-benefit of this procedure. ${ }^{19}$

Outcomes for 38 implant recipients with a Nucleus device were investigated retrospectively. All participants were diagnosed with a bilateral HL at age $\leq 3 \mathrm{yr}$ and were implanted at age $14 \mathrm{yr}$ or older. Nineteen participants had confirmed bilateral, severe to profound HL at age $\leq 12$ mo (prelingual); nine had confirmed bilateral, severe to profound HL at age $>12 \mathrm{mo}$ and $\leq 3 \mathrm{yr}$ (perilingual); and the remaining 10 had a diagnosis of bilateral sensorineural HL at age $\leq 3 \mathrm{yr}$ which progressed to severe to profound HL after 3 yrs of age (progressive). There were 24 females and 14 males, and the average age at implantation was 33 yr (range $=14$ $65 \mathrm{yr}$ ). Closed-set and open-set speech perception tests administered pre- and postimplant were analyzed for all participants, in addition to self-report survey measures of benefit, satisfaction, and implant usage. Participants were placed into one of five hierarchic categories of speech perception performance preimplantation and at 12 mo postimplantation. The categories ranged from sound detection only (category 1) to excellent open-set speech perception (category 5). To be in category 4 or 5 , the participant had to score $>30 \%$ words correct on a recorded version of either the Central Institute for the Deaf Everyday Sentence Lists or the City University of New York Sentences. Before implantation, two recipients (5\%) were in category 4 or 5 compared with $20(53 \%)$ at 12 mo postimplant. Consistent with previous studies, there was large intersubject variability in speech scores. Three factors accounted for $63 \%$ of the variance on open-set sentence test scores, postimplant: Mode of communication in childhood (oral versus total communication/sign), stable as opposed to progressive loss, and time without a hearing aid on the implant ear. More than $80 \%$ of survey respondents used their device $>8 \mathrm{hr}$ a day, and $90 \%$ reported that their ability to understand speech with visual cues was "much better" with the implant. In addition, all reported being satisfied with the device. These results indicate that a cochlear implant should be considered as an option for adults and adolescents with early-onset HL. The majority of participants gained benefit from the device and were satisfied with it. In addition, a substantial number gained good open-set speech perception ability, postimplant. Recipients who used oral communication in childhood, had a progressive loss, and wore a hearing aid on the implant ear up to the time of surgery were more likely to obtain better speech perception outcomes. ${ }^{20}$

Many Canadians are affected by sensorineural hearing loss (SNHL) and those with severe or profound hearing loss may have poor hearing function despite optimized hearing aids. Cochlear implants (CI) offer effective hearing rehabilitation for these patients, however, concern continues to exist regarding possible effects 
of CI on the vestibular system and balance. The objective of this study was to conduct a pilot study assessing the effects of unilateral cochlear implantation (CI) on balance and the vestibular system in post-lingually deafened adults. Twelve patients were included in this pilot study and were assessed pre-operatively and at immediate, 1 week, and 1 month post-operative intervals. Assessments consisted of the dizziness handicap inventory (DHI), subjective visual vertical (SVV), and timed up-and-go testing (TUG). When applicable, testing was repeated with the CI on and off. Many patients were found to have deviated SVV at pre-operative and post-operative assessments. However, statistically significant changes were not seen when comparing pre-operative and postoperative SVV or when comparing SVV with the CI on and with the CI off. DHI was found to improve in five patients and worsen in two patients, however, no statistically significant change was found in DHI scores or with TUG testing. This current pilot study does not indicate that CI surgery or implant activity influence vestibular or balance function, however, this pilot study is underpowered and greater numbers of patients would need be assessed to confirm this findings. ${ }^{21}$

Cochlear implantation (CI) in subjects with unilateral profound sensorineural hearing loss was investigated. The authors of the present study demonstrated the binaural auditory outcomes in a 12- and 36-month prospective cohort outcome study. The present study aimed to do a long-term (LT) evaluation of the auditory outcomes in an analogous study group. LT evaluation was derived from 12 single-sided deaf (SSD) CI recipients and from $11 \mathrm{CI}$ recipients with asymmetric hearing loss (AHL). A structured interview was conducted with each subjects. Speech perception in noise and sound localization were assessed in a $\mathrm{CI}_{\mathrm{OFF}}$ and in a $\mathrm{CI}_{\mathrm{ON}}$ condition. Four binaural effects were calculated: summation effect $\left(\mathrm{S}_{0} \mathrm{~N}_{0}\right)$, squelch effect $\left(\mathrm{S}_{0} \mathrm{~N}_{\mathrm{CI}}\right)$, combined head shadow effect $\left(\mathrm{S}_{\mathrm{CI}} \mathrm{N}_{0}\right)$, and spatial release from masking (SRM). At the LT evaluation, the contribution of a CI or a bone conduction device on speech perception in noise was investigated in two challenging spatial configurations in the SSD group. All (23/23) subjects wore their CI 7 days a week at LT follow-up evaluation, which ranged from 3 to 10 years after implantation. In the SSD group, a significant combined head shadow effect of $3.17 \mathrm{~dB}$ and an SRM benefit of $4.33 \mathrm{~dB}$ were found. In the AHL group, on the other hand, the summation effect $(2.00 \mathrm{~dB})$, the squelch effect $(2.67 \mathrm{~dB})$, the combined head shadow effect $(3.67 \mathrm{~dB})$, and SRM benefit $(2.00 \mathrm{~dB})$ were significant at LT testing. In both the spatial challenging configurations, the speech in noise results was significantly worse in the condition with the bone conduction device compared with the unaided condition. No negative effect was found for the $\mathrm{CI}_{\mathrm{ON}}$ condition. A significant benefit in the $\mathrm{CI}_{\mathrm{ON}}$ condition was found for sound localization compared with the $\mathrm{CI}_{\mathrm{OFF}}$ condition in the SSD group and in the AHL group. All subjects wore their CI 7 days a week at LT follow-up evaluation. The presence of binaural effects has been demonstrated with speech in noise testing, sound localization, and subjective evaluation. In the AHL group, all investigated binaural effects were found to be significant. In the SSD group on the other hand, only SRM and the head shadow, the two most robust binaural effects, were significantly present. However, it took 12M before the SSD and the AHL subjects significantly benefit from the head shadow effect. These reported results could guide counseling of future CI candidates with SSD and AHL in general. ${ }^{22}$

Changes in hearing disability and handicap as evaluated using the Abbreviated Profile of Hearing Aid Benefit (APHAB), the Nijmegen Cochlear Implant Questionnaire (NCIQ), and the Glasgow Benefit Inventory (GBI). Data of 33 patients (mean postoperative duration of $7.5 \mathrm{yr}$ ) were available. No difference in subjective results was found between the VSB and Otologics MET patient groups. Total percentage of nonuse was $13 \%$. Long-term APHAB results show a significant decrease in disability for $43 \%$ of the patients compared with $54 \%$ at 1-year postoperative. NCIQ results show a significant benefit for all subdomains with a negative trend over time. The GBI results show a significant long-term increase in quality of life with positive scores for $82 \%$ of the assessed patients. Long-term postoperative patient satisfaction and quality of life results show a significant difference compared with preoperative measurements, with conventional hearing aids. A negative trend over time is found on all questionnaires, which might reflect patient aging (increase of hearing loss) or habituation to a situation with fewer concerns regarding a patient's external otitis. ${ }^{23}$

Study participants in Luco et al research were assessed using pure tone audiometry, aided speech understanding in quiet (CNC words) and in noise (AzBio sentences at +10 and $+5 \mathrm{~dB}$ SNR) in the sound field with unilateral and bilateral hearing aids fit to target. Participants completed subjective scales of quality of life, (Health Utilities Index Mark 3), hearing disability, (Speech, Spatial and Qualities of Hearing Scale) and a device use satisfaction scale. Participants $\geq 55$ years were administered the Montreal Cognitive Assessment screening tool. One-hundred enrolled individuals completed baseline evaluations. Aided bilateral mean speech understanding scores were $28 \%$ for $\mathrm{CNC}$ words and $31 \%$, and $17 \%$ for AzBio sentences at a $+10 \mathrm{~dB}$, and $+5 \mathrm{~dB}$ SNR, respectively. Mean scale ratings were 0.46 for overall quality of life and 3.19 for functional hearing ability. Ninety percent of participants reported dissatisfaction with overall hearing performance.Evaluation results, including functional performance metrics quantifying the deleterious effects of hearing loss for overall wellbeing, underscore that bilateral hearing aids are not an effective treatment for individuals with bilateral, moderate sloping to profound sensorineural hearing loss. Individuals with this degree of hearing impairment, who demonstrate poor aided speech understanding and dissatisfaction with hearing abilities in everyday life, require 
timely referral to a Cochlear implant clinic for further evaluation. ${ }^{24}$

Ramos et al conducted a study on Ten patients suffering from severe-to-profound sudden hearing loss and tinnitus in the affected ear received implants. The sample comprised 4 men and 6 women, with a mean age of 42.7 years (range 34-62) at implantation. The severity of the tinnitus was evaluated with the Spanish validated version of the Tinnitus Handicap Inventory (THI) and a visual analogue scale. These assessments were obtained before and after implantation. Tinnitus suppression was observed in 2 patients. In 7 cases, we observed an improvement in the THI, in different degrees, and 1 patient remained without changes. Tinnitus worsening was not found in the series studied.Tinnitus reduction following cochlear implantation can be explained by several mechanisms, such as habituation, acoustic masking, direct stimulation of the cochlear nerve and reorganisation of cortical areas. Even though further research is required, cochlear implantation is an effective method for the treatment of disabling tinnitus in patients with severe-to-profound unilateral sudden sensorineural hearing loss. ${ }^{25}$

\section{DISCUSSION}

Cardiovascular risk factors (smoking, increased alcohol consumption) appeared to be associated with a higher risk of developing SSNHL. A low level of serum folate may also be implicated as a risk factor. Factor V Leiden and MTHFR gene polymorphisms were found to occur more frequently in patients with sensorineural hearing loss in several studies, suggesting these inherited prothrombophilic mutations could be independent risk factors of SSNHL. CI performance has improved and more evidence was found for the positive effect of residual hearing on CI outcome, strengthening the growing need for a shift of CI indication. Timely implantation may lead to better speech understanding with a CI. Since hearing loss might deteriorate over time, excluding patients with severe hearing loss will imply a delay rather than an irrevocable refusal for CI. To this end, audiologists and otolaryngologists should be aware of the irreversible consequences of poor speech perception. Poor speech perception leads to poor communication, which has a devastating effect on an individual's quality of life. The speech recognition results are in line with the literature, suggesting that waiting too long before CI might increase the risk of auditory deprivation. It remains debatable what the exact criteria should be to justify both residual hearing on the one side and need for improved hearing on the other side.

\section{CONCLUSION}

Many studies suggested that cochlear implants improve quality of life of receivers and also have a positive effect on social and professional life. In some cases negative outcomes were seen like in a case device failure was observed and also alteration of intellectual abilities. But overall outcomes of cochlear implants were favorable in all aspects from speech recognition to intellectuality.

\section{REFERENCES:}

1. Cruickshanks, K. J., Wiley, T. L., Tweed, T. S., Klein, B. E. K., Klein, R., MaresPerlman, J. A., et al. (1998). Prevalence of hearing loss in older adults in Beaver Dam, Wisconsin: the epidemiology of hearing loss study. Am. J. Epidemiol. 148, 879-886. doi: 10.1093/oxfordjournals.aje.a009713

2. Sindhusake, D., Mitchell, P., Smith, W., Golding, M., Newall, P., Hartley, D., et al. (2001). Validation of self-reported hearing loss. The Blue Mountains Hearing Study. Int. J. Epidemiol. 30, 1371-1378. doi: 10.1093/ije/30.6.1371

3. Amieva, H., Ouvrard, C., Giulioli, C., Meillon, C., Rullier, L., and Dartigues, J.-F. (2015). Self-reported hearing loss, hearing aids, and cognitive decline in elderly adults: a 25-year study. J. Am. Geriatr. Soc. 63, 2099-2104. doi: 10.1111/jgs. 13649

4. Hogan, A., O'loughlin, K., Miller, P., and Kendig, H. (2009). The health impact of a hearing disability on older people in Australia. J. Aging Health 21, 1098-1111. doi: 10.1177/0898264309347821

5. World Health Organization [WHO] (2009). Ageing

6. Swan, W. (2010). Australia to 2050: Future Challenges, ed. D. O. T. Treasury (Canberra: Australian Government).

7. Holden LK, Finley CC, Firszt JB, Holden TA, Brenner C, Potts LG, Gotter BD, Vanderhoof SS, Mispagel K, Heydebrand G, Skinner MW. Factors affecting open-set word recognition in adults with cochlear implants. Ear Hear 2013;34:342-60

8. Carlson ML, Driscoll CL, Gifford RH, Service GJ, Tombers NM, Hughes-Borst BJ, Neff BA, Beatty CW. Implications of minimizing trauma during conventional cochlear implantation. Otol Neurotol. 2011;32:9628.

9. Roeser, R. J., Valente, M. and Hosford-Dunn, H., Audiology diagnosis, 1st ed.Thieme Medical Publishers, New York; 2000.

10. Olivier Sterkers, Isabelle Mosnier, Emmanuelle Ambert-Dahan, Evelyne Herelle-Dupuy, Alexis BozorgGrayeli \& Didier Bouccara (2004) Cochlear Implants in Elderly People: Preliminary Results, Acta OtoLaryngologica, 124:sup552, 64-67, 
11. Angeli SI, Yan D, Telischi F, et al. Etiologic diagnosis of sensorineural hearing loss in adults. Otolaryngology-Head and Neck Surgery. 2005;132(6):890-895. doi:10.1016/j.otohns.2005.03.001

12. Huinck, W.J., Mylanus, E.A.M. \& Snik, A.F.M. Expanding unilateral cochlear implantation criteria for adults with bilateral acquired severe sensorineural hearing loss. Eur Arch Otorhinolaryngol 276, 1313-1320 (2019). https://doi.org/10.1007/s00405-019-05358-Z

13. Greenberg, Simon L.; Shipp, David; Lin, Vincent Y.; Chen, Joseph M.; Nedzelski, Julian M. Cochlear Implantation in Patients With Bilateral Severe Sensorineural Hearing Loss After Major Blunt Head Trauma, Otology \& Neurotology: January 2011 - Volume 32 - Issue 1 - p $48-54$ doi: 10.1097/MAO.0b013e3181ff73fd

14. Sladen, Douglas P.; Carlson, Matthew L.; Dowling, Brittany P.; Olund, Amy P.; DeJong, Melissa D.; Breneman, Alyce; Hollander, Sara; Beatty, Charles W.; Neff, Brian A.; Driscoll, Colin L. Cochlear Implantation in Adults With Asymmetric Hearing Loss: Speech Recognition in Quiet and in Noise, and Health Related Quality of Life, Otology \& Neurotology: June 2018 - Volume 39 - Issue 5 - p 576-581

15. Brian Schwab, Michele Gandolfi, Erica Lai, Erin Reilly, Lorie Singer, Ana H. Kim. The Impact of Age on Cochlear Implant Performance. International Journal of Otolaryngology and Head \& Neck Surgery Vol.04 No.05(2015), Article ID:59282,8 pages

16. Buss E, Dillon MT, Rooth MA, et al. Effects of Cochlear Implantation on Binaural Hearing in Adults With Unilateral Hearing Loss. Trends in Hearing. January 2018. doi:10.1177/2331216518771173

17. Huarte A. ' Lezaun R. ' Manrique M. Quality of Life Outcomes for Cochlear Implantation in the Elderly. Audiol Neurotol 2014;19(suppl 1):36-39.

18. Van Loon, Maarten Caspar, Smits, Cas, Smit, Conrad F, Hensen, Erik F. Merkus, Paul Cochlear Implantation in Adults With Asymmetric Hearing Loss: Benefits of Bimodal Stimulation, Otology \& Neurotology: July 2017 - Volume 38 - Issue 6 - p e100-e106 doi: 10.1097/MAO.0000000000001418

19. Olivier Sterkers, Isabelle Mosnier, Emmanuelle Ambert-Dahan, Evelyne Herelle-Dupuy, Alexis BozorgGrayeli, Didier Bouccara. Cochlear Implants in Elderly People: Preliminary Results. https://doi.org/10.1080/03655230410017184

20. Caposecco, Andrea; Hickson, Louise; Pedley, Karen Cochlear Implant Outcomes in Adults and Adolescents With Early-Onset Hearing Loss, Ear and Hearing: March/April 2012 - Volume 33 - Issue 2 - p 209-220 doi: 10.1097/AUD.0b013e31822eb16c

21. Le Nobel, G.J., Hwang, E., Wu, A. et al. Vestibular function following unilateral cochlear implantation for profound sensorineural hearing loss. J of Otolaryngol - Head \& Neck Surg 45, 38 (2016). https://doi.org/10.1186/s40463-016-0150-6

22. Mertens, Griet; De Bodt, Marc, Van de Heyning, Paul .Evaluation of Long-Term Cochlear Implant Use in Subjects With Acquired Unilateral Profound Hearing Loss: Focus on Binaural Auditory Outcomes, Ear and Hearing: January/February 2017 - Volume 38 - Issue 1 - p 117-125

23. Zwartenkot, Joost W.; Hashemi, Javad; Cremers, Cor W. R. J.; Mulder, Jef J. S.; Snik, Ad F. M. Active Middle Ear Implantation for Patients With Sensorineural Hearing Loss and External Otitis, Otology \& Neurotology: July 2013 - Volume 34 - Issue 5 - p 855-861 doi: 10.1097/MAO.0b013e31828f47c2

24. J. Eric Lupo, Allison Biever, David C. Kelsall,Comprehensive hearing aid assessment in adults with bilateral severe-profound sensorineural hearing loss who present for Cochlear implant evaluation,American Journal of Otolaryngology, Volume 41, Issue 2,2020,102300,ISSN 0196-0709,

25. Ángel Ramos, Rubén Polo, Elisabeth Masgoret, Ovidio Artiles, Isidoro Lisner, María L. Zaballos, Cecilia Moreno, Ángel Osorio, Cochlear Implant in Patients With Sudden Unilateral Sensorineural Hearing Loss and Associated Tinnitus,Acta Otorrinolaringologica (English Edition),Volume 63, Issue 1,2012,Pages 15-20, ISSN 2173-5735 